\title{
Influences of closure, occlusion, and size on the perception of fragmented pictures
}

\author{
JAMES M. BROWN and CHRISTOPHER KOCH \\ University of Georgia, Athens, Georgia
}

\begin{abstract}
Two experiments examined the ability to recognize a repeated figure in stimuli where only fragments of the figures were presented. Fragments were either closed or open regions presented either with or without an occluder that filled the spaces between the fragments (Experiment 1). Recognition performance was significantly better for open fragments, with or without an occluder, contrary to previous assertions that the occluder is the important factor for recognition. When stimulus size was varied, an occluder hindered recognition performance in the smallest size condition relative to unoccluded stimuli, which were equally easy to recognize across all size conditions (Experiment 2). The size results support the idea of size invariance in object naming. The difficulty in the smallest occluded condition is discussed in terms of differences in global and local processing depending on visual angle and sparsity.
\end{abstract}

Stimuli such as those in Figures 1a and 1c have been used in demonstrations and discussions of the Gestalt organizational principles of closure and Pragnanz (Bregman, 1981, 1990; Pomerantz \& Kubovy, 1981). In Figure 1c, interposition information from the occluding surface "accounts for" the missing portions of the Bs, allowing closure of the local fragments into recognizable figures (Bregman, 1981). In Figure 1a, one might infer that the Bs are there, but the perceptual experience is not the same. What is it about the fragments in stimuli such as Figure la that makes them difficult to organize into global, recognizable forms? It has been suggested that the missing occluder and the interposition information associated with it prevent closure of the fragments into global forms (Bregman, 1981).

Along the borders where the occluder intersects the Bs in Figure 1c, a physical edge is created that is assigned to, or associated with, the occluder. Phenomenally, the Bs have no edges at these intersections because they are seen as amodally continuous in depth behind the occluder. If the occluder were peeled away in Figure 1c, one would probably be surprised to see Figure 1a. Besides removing the occluder to create Figure 1a from Figure 1c, contours must be added to completely enclose the fragments. These contours bias the organization of the fragments as separate, enclosed regions. Thus, closure is working against a global organization. The extra lines are irrelevant to the global forms requiring the viewer to make a distinction between the relevant and irrelevant features of the stimulus (Kennedy, 1974).

Correspondence should be addressed to J. M. Brown, Department of Psychology, University of Georgia, Athens, GA 30602-3013 (e-mail: jmbrown@uga).

\section{EXPERIMENT 1}

We were interested in examining whether the inability to identify any global forms in stimuli such as Figure 1a is due to the physical closure of the fragments or the absence of the occluder's interposition information. To test this idea, we created open fragment stimuli (e.g., Figures $1 \mathrm{~b}, \mathrm{e}$, and $1 \mathrm{f}$ ), without an occluder and without any additional lines, and closed fragment stimuli that combined the closed fragments with an occluder (e.g., Figure 1d).

If occlusion information is critical for organizing the fragments into global forms, then the repeated figures should be easier to recognize in the occluded stimuli than in the unoccluded stimuli regardless of whether or not the fragments are open or closed. Thus, recognition of the repeated forms in stimuli such as Figures $1 \mathrm{c}$ and $1 \mathrm{~d}$ should be better than for stimuli such as Figures $1 \mathrm{a}$ and $1 \mathrm{~b}$. On the other hand, if closure of the fragments (i.e., open vs. closed fragments) is the critical factor affecting recognition of the global forms, then open fragment stimuli should lead to better recognition than should closed fragment stimuli regardless of whether or not the occluder is present. Thus, recognition of the repeated forms in stimuli such as Figures $1 \mathrm{~b}$ and $1 \mathrm{c}$ should be better than for stimuli such as Figures 1a and 1d.

\section{Method}

Subjects. Thirty-two observers with normal or corrected-tonormal vision participated for credit in their introductory psychology course.

Stimuli. Two additional stimuli were constructed similar to the Bs (Figures 1a-1d): the letter $A$ and the female symbol (open versions shown in Figures $1 \mathrm{e}$ and 1f, respectively). To maintain consistency with the Bs stimuli, the $A$ and female symbol stimuli were matched on several dimensions. The As and female signs making up the stimuli (1) had five exact replicas in various orientations, (2) were the same size, and (3) had the same percent area occluded 


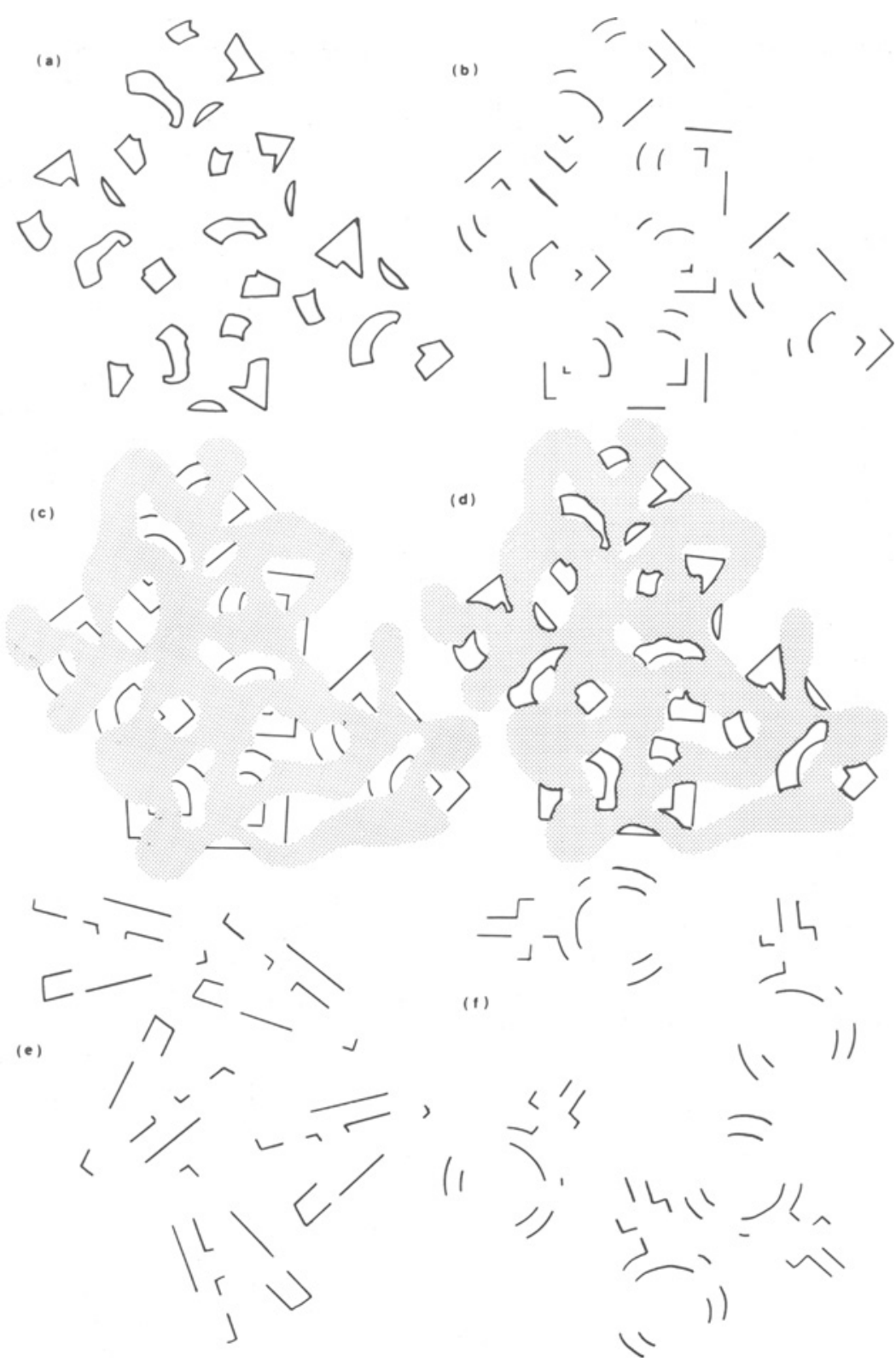

Figure 1. Examples of (a) closed/unoccluded, (b, e, f), open// unoccluded, (c) open/occluded, and (d) closed/occluded stimuli used in Experiment 1.

as the Bs. Identifying characteristics were also taken into account. According to Gibson's (1969) list of distinctive features for letter recognition, approximately half were eliminated in the occluded Bs stimulus. In an effort to construct a set of comparable stimuli, the number and type of distinctive features were likewise manipulated in the $A$ and female symbol. Each stimulus display subtended $11.75^{\circ}$ (height [h]) $\times 16.47^{\circ}$ (width [w]) visual angle at the viewing distance of $40 \mathrm{~cm}$. Each stimulus item subtended $5.87^{\circ}$ (h) $\times$ $3.58^{\circ}(w)$ and was constructed of black lines $9^{\prime}$ thick on a white background. The occluder was a stippled gray region.

Procedure. A $2 \times 2$ between-subject design was used, with subjects randomly assigned to one of four experimental groups. Stimuli varied according to (1) fragment type (open or closed) and (2) occlusion information (occluder present or absent). Thus, the four experimental groups were: closed fragments/occluder absent (e.g., Figure 1a), closed fragments/occluder present (e.g., Figure 1d), open fragments/occluder absent (e.g., Figures $1 \mathrm{~b}$, le, and If), and open fragments/occluder present (c.g., Figure 1c). Pilot studies revealed significant carryover or set effects from viewing one stimulus type to another; therefore, only one stimulus type was presented to each experimental group.

The subjects were told that they were about to be presented with three pictures, each having a different repeated figure in it. They were instructed to identify the repeated figure as quickly as possible. They were told they would have $1 \mathrm{~min}$ to correctly identify the repeated figure. They were instructed to keep guessing what they thought the repeated figure was, until the experimenter told them that they had correctly identified the repeated figure or when 
the 1-min trial was over. All responses were recorded. The dependent measure was time elapsed between stimulus presentation and correct identification of the repeated figure with a 1-min limit (i.e., subjects unable to identify the repeated figure were given an identification time of $60 \mathrm{sec}$ ). Thus, initial guesses that were incorrect were noted but the trial was continued until either the repeated figure was correctly identified or the 1-min trial had elapsed. Stimuli were presented in a pseudorandom letter-symbol-letter order. Either the A or the B was presented first followed by the female symbol and then the other letter. Stimuli were presented as flip charts.

\section{Results}

A 2 (closed vs. open fragment type) $\times 2$ (occluded or unoccluded) analysis of variance (ANOVA) of identification times was significant $[F(3,28)=31.26, p<$ $.0001]$. Only the main effect of fragment type was significant $[F(1,28)=91.37, p<.0001]$. Open fragment stimuli were identified faster and correctly identified more often $\left[\chi^{2}(1)=21.89, p<.0001\right]$ than were closed fragment stimuli, whether or not occlusion information was present. Mean identification times for the four conditions are summarized in Table 1.

Mean identification times for open fragment/occluder present and occluder absent stimuli were 3.78 and $4.15 \mathrm{sec}$, respectively. Closed fragment stimuli produced longer response times with occluder present and absent conditions requiring 38.28 and $48.58 \mathrm{sec}$, respectively. The results suggest occlusion information was not critical for identifying the global figures from the local fragments. Although we knew identification of the global forms was possible with occlusion information present (e.g., open fragments/occluder present), the results show that it was not necessary (e.g., open fragments/occluder absent).

\section{Discussion}

What is it about the physical closure of the fragments that makes them difficult to organize into global figures? From a figure-ground perspective, this may occur because the enclosed fragments in stimuli such as Figure 1a are segregated as small figure regions surrounded by a white background (i.e., Rubin's, 1921/1958, principle of surroundedness). These small regions are perceptually complete; therefore, there is no tendency to process them further as parts of larger figures. The same could be argued for stimuli such as Figure 1d, with the gray surface interspersed between the enclosed fragments also con-

Table 1

Mean Identification Times $( \pm S E)$ for Experiment 1 Conditions

\begin{tabular}{cccccccc}
\hline & \multicolumn{4}{c}{ Fragment Type } \\
\cline { 2 - 4 } \cline { 5 - 7 } Occluder & $M$ & $S E$ & $n$ & & $M$ & $S E$ & $n$ \\
\hline Present & 48.58 & 16.35 & 1 & 4.15 & 1.19 & 8 \\
Absent & 38.28 & 11.99 & 2 & & 3.78 & 1.84 & 8 \\
\hline
\end{tabular}

Note $-M=$ mean identification time, expressed in seconds. $S E=$ standard error $( \pm), n=$ number of subjects (out of 8 ) who correctly identified all three stimuli. tributing to their segregation. The ability to see the open fragments as parts of global forms in stimuli such as Figure 1c could be explained in terms of the perception of the occluder as figure in front of the missing portions. Although the fragments are physically incomplete, they are perceptually completed behind the occluder (as Bregman, 1981, 1990, has suggested). However, stimuli such as Figures $1 \mathrm{~b}, 1 \mathrm{e}$, and $1 \mathrm{f}$ are exceptions to this figureground interpretation. There is no occluding surface (real or illusory ${ }^{1}$ ) behind which the physically incomplete fragments can be interpreted to complete. Still, we are able to perceptually extrapolate from the lines that are present to form a representation of the global forms. We would argue that phenomenally this is a different type of perceptual completion. In Figure 1c, the forms are amodally complete, but not in Figures 1b, 1e, and 1f.

It may be informative to consider this experiment as a study in picture perception. Such a perspective focuses attention on such stimuli as depictions that differ in what they represent to the viewer. Kennedy (1974) suggests relevant features must be distinguished from the "irrelevant rubble" when viewing incomplete pictures. According to this view, the extra lines added to enclose the fragments in Figures $1 \mathrm{a}$ and $1 \mathrm{~d}$ are irrelevant lines to the completion of the global forms. The global forms in Figures $1 \mathrm{~b}$ and $1 \mathrm{c}$ may be identified easier than Figures la and 1d because there are no extra lines to process and distinguish as irrelevant. However, while the global forms in Figures $1 \mathrm{~b}$ achieve representational completion, as evidenced by their recognition, they never achieve perceptual completion in the same sense that they do in Figure 1c.

Nakayama, Shimojo, and Silverman (1989) have asked a similar question about stimuli such as Figures 1a and 1c: "What is it about the visibility of the occluder that enables the occluded objects to be more easily recognized?" They propose that contours created on the retina by the overlap of objects in depth are classified by the visual system as intrinsic to the occluding object while at the same time being classified as extrinsic to the occluded object. They define intrinsic contours as part of the bounding contours of an object that contribute to object identification and extrinsic contours as extraneous edges with no relation to object identification. According to this view, we are able to see the global form in Figure 1c because the borders between the occluder and the fragments are classified as intrinsic to the occluder and extrinsic to the fragments on the basis of depth information. Once this depth-based edge classification occurs, the amodal completion of the fragments behind the closer occluder follows. In Figure 1a, depth information is absent; thus, the contours previously classified as extrinsic to the fragments are now classified as intrinsic to them precluding their interpretation as incomplete parts of more global forms. A similar explanation would hold for stimuli such as Figure 1d, where the fragments can be seen as behind, in the same depth plane as, or in front of the 
occluder. However, this theory also does not adequately account for the phenomenological experience of organizing stimuli such as Figures $1 \mathrm{~b}, 1 \mathrm{e}$, and $1 \mathrm{f}^{2}$

Another perspective on these stimuli and results comes from a recent theory of visual interpolation in object perception particularly concerned with situations where boundaries are formed across uniform regions of space and where spatially separated areas are perceived to be connected (Kellman \& Shipley, 1991). A detailed account of their theory is beyond the scope of this paper, but the three major guidelines of the theory are particularly relevant here: (1) discontinuities in space or time are necessary for visual interpolation; (2) new contours are perceived when the edges leading into discontinuities are relatable to others, with relatability depending on relations in local edge orientations; (3) a new unit is formed when connected edges enclose an area. The difficulty seeing global forms in stimuli such as Figures la and 1d would be due to the closed fragments being classified as individual units, thus eliminating any discontinuities that might be used to interpolate across the space between them. The classification of the closed fragments as individual units may be due to the accidental $L$ vertices formed by the additional lines enclosing the fragments (Blickle, 1989; Hummel \& Biederman, 1992). In Figure 1c, the discontinuities formed where the lines of the open fragments meet the occluder allow for visual interpolation. Due to the brightness difference between the occluder and the lines, new contours are amodally perceived where the interrupted edges are relatable to other interrupted edges. The same explanation involving discontinuities and relatable edges could be used for Figures $1 \mathrm{~b}, 1 \mathrm{e}$, and $1 \mathrm{f}$, except the completed forms result in neither modally or amodally perceived edges. In other words, the theory seems to account for why unit formation processes might group the regions in Figures $1 b, 1 e$, and $1 \mathrm{f}$ into global forms, but it does not offer an explanation for the particular perceptual experience. The theory does propose, however, that the perceptual outcome of the unit formation processes (e.g., whether or not interpolation occurs, and whether it is modal, amodal, etc.) is intimately linked with available depth information about the units.

\section{EXPERIMENT 2}

Considering that the occluder had no effect on recognition performance in Experiment 1, does this mean open/occluded and open/unoccluded stimuli would always be processed equally well? Would the ability to extract the important figural information from such images be affected if the information were concentrated over a smaller region of the retina? Research involving other perceptual tasks has shown that retinal size can influence perceptual processing. Large and small versions of the same stimuli are processed differently. For example, in studies of global precedence, larger stimuli were processed faster (Navon \& Norman, 1983). In a study on the perception of ambiguous figures, the interpretation of large figures was more easily influenced by previous information than was the interpretation of smaller versions of the figures (Goolkasian, 1991). These results suggest that larger stimuli may have a processing advantage over relatively smaller stimuli. Would this processing advantage also be found when the task is to organize incomplete stimuli into recognizable wholes?

A number of previous studies investigating object recognition in children and adults have used fragmented or incomplete pictures of objects (i.e., what we have called "open/unoccluded stimuli'). However, most of these studies were concerned with how object recognition is affected by the amount of image contour deleted and the number and type of distinctive features present (Blickle, 1989; Gollin, 1960, 1961, 1962; Murray \& Kinnison, 1989; Murray \& Szymczyk, 1978). In addition, the majority of these studies have used stimuli of only one size, and that size has been rather large. For example, some individual stimulus sizes used were $13.34^{\circ} \times 19.57^{\circ}$ by Murray and Szymczyk $(1978), 26.6^{\circ} \times 16.7^{\circ}$ by Murray and Kinnison (1989), and approximately $30^{\circ} \times 21^{\circ}$ by Gollin (1960). The one study that has varied the size of their incomplete stimuli while examining recognition performance used stimuli that ranged from $3.77^{\circ}$ to $5.78^{\circ}$ over a series of experiments (Blickle, 1989). In general, as stimulus size increased from $3.77^{\circ}$ to $4.75^{\circ}$ mean correct reaction time decreased, but then increased beyond the smallest stimuli reaction times as size increased further from $4.95^{\circ}$ to $5.78^{\circ}$ (Blickle, 1989). These findings, and the previous results showing influences of retinal size on other aspects of perceptual processing, suggest that the recognition of fragmented figures would be influenced by their size. Smaller stimuli should be more difficult to recognize than larger versions.

On the other hand, the results of a recent study on naming reaction time for complete objects (Biederman \& Cooper, 1992) suggest that the identification of fragmented figures may not be influenced by the size of the stimuli. Pictures of objects were presented in a priming task. In one of the conditions, the size of the initially viewed objects (i.e., the primes) were smaller than, the same size as, or larger than the same objects viewed later (i.e., the targets). Subjects had to name the target objects as fast as possible. Naming reaction times were independent of the prime-target size relation. We are asking subjects to identify (i.e., name) the repeated figures in our stimuli. If naming fragmented figures is utilizing the same processes as those used in naming complete objects, then we should find no difference in identification times as the sizes of the fragmented stimuli are varied. To examine the effect of retinal size on the organization of our fragmented stimuli, we compared recognition performance for open/occluded and open/unoccluded stimuli such as those used in Experiment 1 as a function of retinal size.

\section{Method}

Subjects. Fifty-six observers with normal or corrected-to-normal vision participated for credit in their introductory psychology course.

Stimuli. All stimuli contained three repeated figures (As, Bs, or 8s). A small and a large version of each stimulus was created. The overall dimensions of the small versions were $3.13 \mathrm{~cm}(\mathrm{~h}) \times$ 
$3.07 \mathrm{~cm} \mathrm{(w),} \mathrm{with} \mathrm{individual} \mathrm{figures} 1.87 \mathrm{~cm}(\mathrm{~h}) \times 1.37 \mathrm{~cm}(\mathrm{w})$. Large versions were $15.83 \mathrm{~cm}(\mathrm{~h}) \times 16.17 \mathrm{~cm}(w)$, with the individual figures $6.93 \mathrm{~cm} \mathrm{(h)} \times 5.6 \mathrm{~cm}(\mathrm{w})$. Both large and small versions of all stimuli (i.e., occluded and unoccluded) were presented at two viewing distances, 70 and $92.5 \mathrm{~cm}$, creating four size conditions. The size of the small stimuli at the $92.5-\mathrm{cm}$ viewing distance (Size 1) was $1.90^{\circ}(\mathrm{h}) \times 1.94^{\circ}(\mathrm{w})$, with individual stimuli subtending $0.85^{\circ}(\mathrm{h}) \times 0.68^{\circ}(\mathrm{w})$. The size of the small stimuli at the $70-\mathrm{cm}$ viewing distance (Size 2) was $2.51^{\circ}(\mathrm{h}) \times 2.56^{\circ}(\mathrm{w})$, with individual figures subtending $1.12^{\circ}(\mathrm{h}) \times 0.90^{\circ}(\mathrm{w})$. The size of the large stimuli at the $92.5-\mathrm{cm}$ viewing distance (Size 3) was $9.71^{\circ}(\mathrm{h}) \times 9.91^{\circ}(\mathrm{w})$, with individual stimuli subtending $4.29^{\circ}(\mathrm{h})$ $\times 3.46^{\circ}(w)$. The size of the large stimuli at the $70-\mathrm{cm}$ viewing distance (Size 4) was $12.75^{\circ}(\mathrm{h}) \times 13.0^{\circ}(\mathrm{w})$, with individual figures subtending $5.67^{\circ}(\mathrm{h}) \times 4.57^{\circ}(\mathrm{w})$. All figures were made of 1 -mm-thick black lines on a white background. The occluder was black.

Design. A $2 \times 4$ between-subject factorial design was utilized, with identification time serving as the dependent measure. Stimuli varied according to occlusion (occluded or unoccluded) and size (four sizes).

Procedure. The procedure was the same as that for Experiment 1, except the stimuli were presented on cards that the experimenter uncovered each trial. The subjects first saw the As followed by the $8 \mathrm{~s}$ followed by the Bs, or the reverse order. Presentation order was counterbalanced across conditions.

\section{Results and Discussion}

A 2 (occlusion) $\times 4$ (size) ANOVA on mean identification times was significant for the main effects of occlusion $[F(1,104)=9.62, p<.002]$ and size $[F(3,104)=5.38, p<.002]$. The interaction was not significant $[F(3,104)=2.08, p>.10]$. Mean identification times for the different size conditions are plotted in Figure 2 as a function of occlusion.

Tukey's multiple comparison tests showed the occluded/ Size 1 condition had significantly longer identification times than all other occlusion $\times$ size combinations $(p<$ .05 ) except the occluded/Size 2 condition. Thus, small

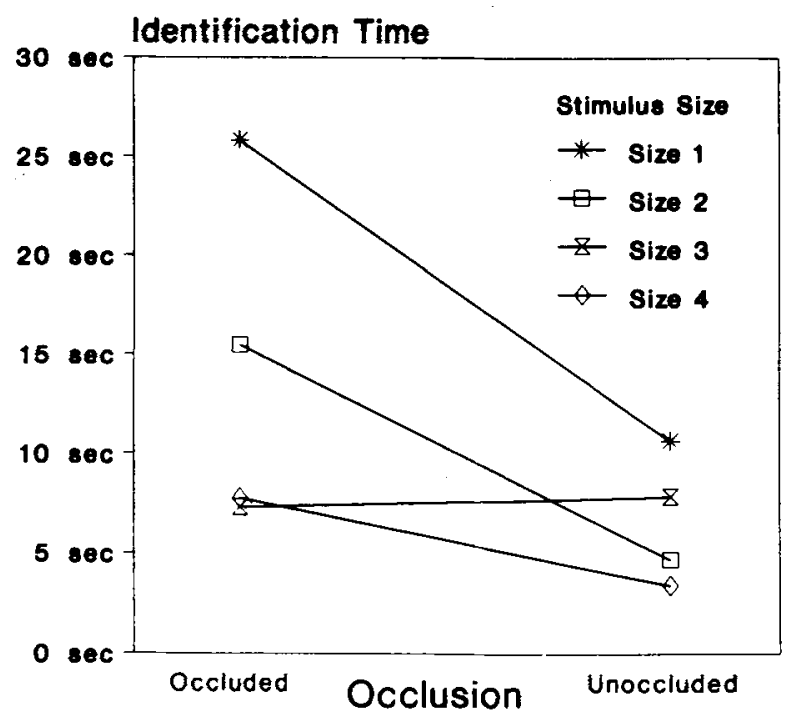

Figure 2. Mean identification time (in seconds) as a function of size and occlusion condition. stimuli were more difficult to organize than large stimuli only when the occluder was present. What was it about the occluder that made it so much more difficult for the subjects to organize the fragments into recognizable wholes when the stimuli were small? If it was a signalto-noise problem-that is, a problem in determining what contours belonged to the occluder and what were the fragments-then a practice trial or two may have been beneficial for the subjects, allowing them to cue into the appropriate information. To examine this possibility, two additional analyses were made. In the first analysis, the first trial for each subject was eliminated and considered a practice trial. Again, the main effects of occlusion $[F(1,104)=9.30, p<.003]$ and size $[F(3,104)=6.48$, $p<.0001]$ were significant, but now the interaction was also significant $[F(3,104)=3.82, p<.01]$. The second analysis eliminated the first two trials as practice and also found significant main effects of occlusion $[F(1,104)=7.18, p<.009]$ and size $[F(3,104)=4.96$, $p<.003]$ and their interaction $[F(3,104)=4.13, p<$ $.008]$. In addition to the significant interaction between occlusion and size after one or two practice trials, the other difference between these analyses and the first was that the post hoc tests (i.e., Tukey's multiple comparison tests) now indicated the occluded/Size 1 condition had significantly longer identification times than all other conditions $(p<.05)$. Although practice naturally resulted in progressively shorter identification times for all conditions, it could not eliminate the debilitating effect of the occluder for recognition in the smallest size (Size 1) condition (see Figure 3).

The combined outcome of these analyses demonstrates three things. First, size did not influence the ability to organize the fragmented figures into recognizable wholes. A 45-fold difference in area between our smallest and largest conditions did not affect performance when the stimuli were unoccluded. This supports the finding of size invariance in complete object naming (Biederman \& Cooper, 1992). However, size did have an effect on performance when an occluder was present. Second, the analyses support previous findings that recognizability of fragmented figures is facilitated with practice (Gollin, 1962; Murray \& Szymczyk, 1978). Once subjects were cued into the general type and size of the repeated figure they were looking for on the first trial, the faster they were able to organize the fragments into complete figures on subsequent trials. Finally, even after two practice trials, the smallest occluded condition (occluded/Size 1) remained difficult. This is in contrast to the unoccluded/ Size 1 condition, which was never significantly different from any other occlusion $x$ size condition. The fact that the occluder can actually hinder organization of the fragments when the stimuli are sufficiently small is particularly interesting given the theoretical considerations concerning the relationship between the occluder and the fragments discussed in Experiment 1 . The concentration of contour and/or pictorial information within a small area on the retina might have led to the difficulty in separating out the "irrelevant rubble" from the important contour 


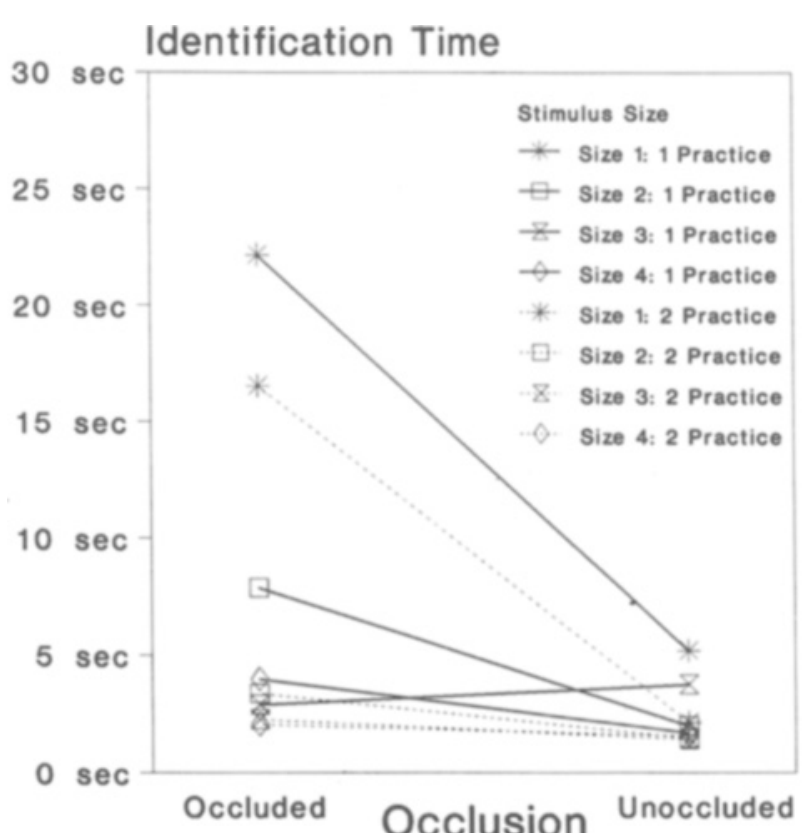

Figure 3. Mean identification time (in seconds) as a function of size and occlusion minus the first trial (solid lines) and the first two trials (dotted lines) as practice.

information (Kennedy, 1974; i.e., decreasing the signalto-noise ratio).

\section{GENERAL DISCUSSION}

In two experiments, we examined the recognizability of global forms when only fragments of the forms were present. Our reason for Experiment 1 was to ascertain whether the inability to identify global forms in stimuli such as Figure 1a is due to the physical closure of the fragments or the absence of the occluder's interposition information. The results showed that physical closure of the fragments was detrimental to recognition whereas absence of the occluder was not. The importance of these results relative to current theories on visual completion and $o c$ clusion information was discussed above. At present, Kellman and Shipley's (1991) theory of visual interpolation in object perception gives the best theoretical account for these results. While not a complete account, it does seem to offer the best arguments for why the image components in the open and closed conditions were grouped differently and why the open/occluded and open/unoccluded conditions were equally easy to perceptually complete.

In Experiment 2, we found that size had no effect on the ability to organize open/unoccluded fragmented figures into recognizable wholes. However, when an occluder was present, size did have an effect on recognition, but only for the smallest size (Size 1) condition. Although the only other study examining the recognition of fragmented figures while varying stimulus size did find an influence of size on mean correct reaction time for single open/ unoccluded stimuli (Blickle, 1989), procedural differences between that study and ours make it difficult to make many direct comparisons. For example, their subjects knew they would be presented single stimuli for brief exposures followed by a mask. Our subjects were instructed to identify the repeated figure in our stimuli without knowing how many times it was repeated. The stimuli were presented under free-viewing conditions, the subjects had up to $1 \mathrm{~min}$ to indicate a correct response, and the trial was not terminated with an incorrect guess. Despite these differences, both experiments found similar results in that subjects were always able to recognize the open/unoccluded fragmented stimuli independent of size. These results are consistent with the finding of size invariance in complete object naming (Biederman \& Cooper, 1992).

What was it about the smallest occluded stimuli that made them so difficult to recognize? Difficulty in resolving the fragments themselves can be ruled out, considering the relative ease of seeing and organizing the fragments in the smallest unoccluded condition. Indeed. when one looks at the smallest occluded stimuli, the fragments are easily visible; however, phenomenally, it is not as immediately obvious how many repeated figures there are. One sees the fragments, but it is not clear how they go together. In the smallest unoccluded condition, one is immediately aware that the fragments group together into three local regions. The transition from a global to a local level of analysis occurs automatically and effortlessly. The transition to the appropriate local (figure) level of processing appears to have been slowed by the presence of an occluder. In other words, the occluder made it more difficult to move from the global level of analysis involved in segregating the fragments from the occluder to a local level of analysis that would have segregated the fragments into local groups that belonged together.

This interpretation is supported by studies examining global and local processing as a function of visual angle (Kinchla \& Wolfe, 1979; Lamb \& Robertson, 1990; Navon \& Norman, 1983) and sparsity (Martin, 1979). In general, the global level of a stimulus has priority over, and can interfere with, processing of the local level when the information is concentrated (Martin, 1979) over a small visual angle (Kinchla \& Wolfe, 1979; Lamb \& Robertson, 1990; Navon \& Norman, 1983). For the larger occluded stimuli, relatively larger spatial scale (e.g., spatial frequency) mechanisms would be involved in processing the occluder, relative to those processing the narrower fragments (Ginsburg, 1976; Graham, 1981; Kinchla \& Wolfe, 1979). This would have allowed the two global aspects of the display to be separated easily. The outputs from these different scale mechanisms could then have differentially fed into interpolative mechanisms. Endstopped outputs from the smaller scale mechanisms could then be related to each other (Grossberg, 1987; Kellman \& Shipley, 1991), thus creating the possibility for interpolation and perceptual completion. However, as the stimuli became smaller, they reached a point where there was relatively less of a difference in scale (i.e., spatial fre- 
quency) between the mechanisms operating on the occluder and those operating on the fragments. In other words, the activity due to the fragments was no longer as easily distinguished from the activity due to the occluder. This paradox never occurred for the unoccluded stimuli as their size decreased.

Considering the relative activity of spatial mechanisms operative under luminance and equiluminance conditions and their proposed contributions to depth perception (Livingstone \& Hubel, 1988), we are currently examining fragmented figure perception with or without an occluder depending on the size of the stimuli and the luminance, color, and lightness relations between the fragments, the occluder, and the background.

\section{REFERENCES}

Biederman, 1., \& CoOper, E. E. (1992). Size invariance in visual object priming. Journal of Experimental Psychology: Human Perception \& Performance, 18, 121-133.

BLICKLE, T. W. (1989). Recognition of contour deleted images. Unpublished doctoral dissertation, State University of New York at Buffalo.

Bregman, A. S. (1981). Asking the "what for" question in auditory perception. In M. Kubovy \& J. R. Pomerantz (Eds.), Perceptual organization (pp. 99-118). Hillsdale, NJ: Erlbaum.

Bregman, A. S. (1990). Auditory scene analysis: The perceptual or ganization of sound. Cambridge, MA: MIT Press.

GiBson, E. J. (1969). Principles of perceptual learning and development. Englewood Cliffs, NJ: Prentice-Hall.

GinsBURG, A. (1976). The perception of visual form: A two-dimensional filter analysis. In V. D. Gleger (Ed.), Proceedings of the Fourth Symposium on Sensory System Physiology: Information Processing in the Visual System. Leningrad: Russian Academy of Sciences.

Gollin, E. S. (1960). Developmental studies of visual recognition of incomplete objects. Perceptual \& Motor Skills, 11, 289-298.

Gollin, E. S. (1961). Further studies of visual recognition of incomplete objects. Perceptual \& Motor Skills, 13, 307-314.

Gollin, E. S. (1962). Factors affecting the visual recognition of incomplete objects: A comparative investigation of children and adults. Perceptual \& Motor Skills, 11, 289-298.

GoOLKaSIAN, P. (1991). The effect of size on the perception of ambig uous figures. Bulletin of the Psychonomic Society, 29, 161-164.

Graham, N. (1981). The visual system does a crude Fourier analysis of patterns. In S. Grossberg (Ed.), Mathematical psychology and psychophysiology. Providence, RI: American Mathematical Society.

GrossberG, S. (1987). Cortical dynamics of three-dimensional form, color, and brightness perception: II. Binocular theory. Perception \& Psychophysics, 41, 117-158.

Hummel, J. E., \& Biederman, I. (1992). Dynamic binding in a neu ral network for shape recognition. Psychological Review, 99, 480-517.

KENNEDY, J. M. (1974). A psychology of picture perception: Images and information. San Francisco: Jossey-Bass.
Kellman, P. J., \& SHiPley, T. F. (1991). A theory of visual interpolation in object perception. Cognitive Psychology, 23, 14I-221.

KinCHLA, R. A., \& WolfE, J. M. (1979). The order of visual processing: "Top-down," "bottom-up," or "middle-out." Perception \& Psychophysics, 25, 225-231.

LAmb, M. R., \& Robertson, L. C. (1990). The effect of visual angle on global and local reaction times depends on the set of visual angles presented. Perception \& Psychophysics, 47, 489-496.

Livingstone, M., \& Hubel, D. (1988). Segregation of form, color, movement, and depth: Anatomy, physiology, and perception. Science, 240, 740-749.

MarTin, M. (1979). Local and global processing: The role of sparsity. Memory \& Cognition, 7, 476-484.

Murray, F. S., \& KinNison, E. L. (1989). Degree of fragmentation and number of distinctive features in the recognition of pictured objects by children and adults. Bulletin of the Psychonomic Society, 27 121-124.

Murray, F. S., \& SzymczyK, J. M. (1978). Effects of distinctive features on recognition of incomplete pictures. Developmental Psychology, 14, 356-362.

Nakayama, K., Shimojo, S., \& Silverman, G. H. (1989). Stereoscopic depth: Its relation to image segmentation, grouping, and the recognition of occluded objects. Perception, 18, 55-68.

Navon, D., \& Norman, J. (1983). Does global precedence really depend on visual angle? Journal of Experimental Psychology: Human Perception \& Performance, 9, 955-965.

Pomerantz, J. R., \& Kubovy, M. (1981). Perceptual organization: An overview. In M. Kubovy \& J. R. Pomerantz (Eds.), Perceptual organization (pp. 423-456). Hillsdale, NJ: Erlbaum.

Rubin, E. (1958). Figure and ground. In D. C. Beardslee \& M. Wertheimer (Eds.), Readings in perception (pp. 194-203). Princeton, NJ: Van Norstrand. (Original work published 1921)

\section{NOTES}

1. After open/unoccluded trials in Experiments 1, the subjects were shown two examples of illusory triangles (e.g., a white-and-black version) and asked several follow-up questions concerning their phenomenological experience. No subjects ever reported seeing an illusory white occluder. The stimuli were always reported as black lines on a white background. After open/unoccluded trials in Experiment 2, the subjects were shown the same two illusory contour triangles while illusory nature of the contours and surfaces were described to them. All subjects rated the visibility of the illusory contours and surfaces of the triangles a 7 on a 7 -point scale $(1=$ never visible, $7=$ always visible $)$. The open/unoccluded stimuli were then shown again one at a time while the subjects rated the visibility of any illusory occluder they might have seen blocking out the figures when they first viewed the stimuli. All subjects gave a rating of 1 .

2. Fragments made of lines were used instead of filled regions, because filled fragments would have eliminated from consideration the extrinsic contours we were interested in.

(Manuscript received April 30, 1992; revision accepted for publication October 6, 1992.) 